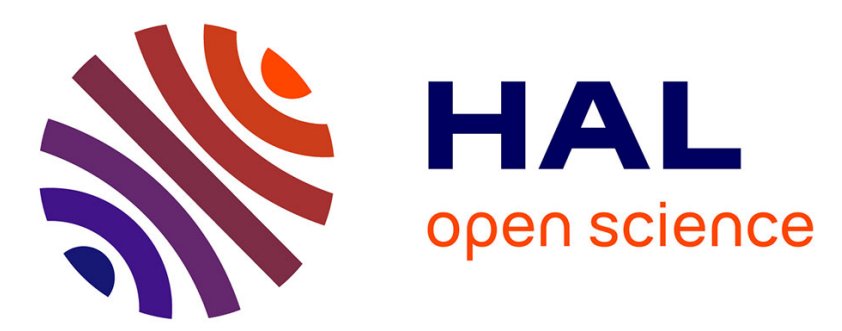

\title{
A homology model for methionyl tRNA synthetase: active site analysis and docking interactions
}

Ehab Al-Moubarak, Claire Simons

\section{To cite this version:}

Ehab Al-Moubarak, Claire Simons. A homology model for methionyl tRNA synthetase: active site analysis and docking interactions. Journal of Molecular Modeling, 2010, 17 (7), pp.1679-1693. 10.1007/s00894-010-0871-9 . hal-00637459

\section{HAL Id: hal-00637459 \\ https://hal.science/hal-00637459}

Submitted on 2 Nov 2011

HAL is a multi-disciplinary open access archive for the deposit and dissemination of scientific research documents, whether they are published or not. The documents may come from teaching and research institutions in France or abroad, or from public or private research centers.
L'archive ouverte pluridisciplinaire HAL, est destinée au dépôt et à la diffusion de documents scientifiques de niveau recherche, publiés ou non, émanant des établissements d'enseignement et de recherche français ou étrangers, des laboratoires publics ou privés. 


\title{
A homology model for Clostridium difficile methionyl tRNA synthetase: active site analysis and docking interactions
}

Received: 11.08.2010 / Accepted: 27.09.2010

Ehab Al-Moubarak and Claire Simons ${ }^{\bowtie}$

Medicinal Chemistry, Welsh School of Pharmacy, Cardiff University, King Edward VII Avenue, Cardiff CF10 3NB, UK

${ }^{\circledR}$ E-mail: simonsc@ cardiff.ac.uk

\begin{abstract}
Treatment of $C$. difficile infection is one of the most difficult biomedical challenges. To develop novel antibacterials, researchers have been targeting bacterial molecular functions that are essential for its growth. The methionyl tRNA synthetase (MetRS) is strictly required for protein biosynthesis and success was reported in developing antibacterials to inhibit this enzyme. The present study was aimed at building and analyzing a homology model for $C$. difficile MetRS in the context of drug design.

A homology model of $C$. difficile MetRS was constructed using Molecular Operating Environment (MOE) software. A. aeolicus MetRS was the main template while the query zinc binding domain was modeled using T. thermophilus MetRS. The model has been assessed and compared to its main template (Ramachandran, ERRAT and ProSA). The active site of the query protein has been predicted from its sequence using a detailed conservation analysis (ClustalW2). Using MOE software, suitable ligands were docked in the constructed model, including a $C$. difficile MetRS inhibitor REP3123 and the enzyme natural substrate, and the key active site residues and interactions were identified. These docking studies have validated the active site conformation in the constructed model and identified binding interactions.
\end{abstract}

Keywords Clostridium difficile $\cdot$ Methionyl tRNA synthetase $\cdot$ Homology model $\cdot$ MOE $\cdot$ Molecular docking 


\section{Introduction}

Clostridium difficile ( $C$. difficile) is the most common cause of healthcare related diarrhoea in hospitals and health care institutions. Hospitalized patients on broad spectrum antibiotic treatment are considered those who are particularly at risk from developing the disease, an example is patients suffering from spinal cord injury who are subject to frequent antibiotic exposure and are hospitalized or using hospital facilities for long periods [1]. It is estimated there has been a 40 percent increase in the $C$. difficile infection (CDI) incidence in the past two years [2] and news sources have shown that in 2008 8,324 people in England and Wales died while affected by the disease, with CDI the cause of death in $50 \%$ of cases [3]. This figure shows a four times increase in the deaths associated with CDI compared with 2001 [3].

C. difficile infection is currently treated with a range of therapies with meronidazole, routinely used as initial therapy for CDI [2]. Discontinuation of antibiotic treatment can restore the gut normal flora and stop the $C$. difficile overgrowth that results in the disease incident [2]. Nevertheless this is not always feasible especially when the patient has been treated for a serious condition, moreover, in the best situations only about 20-25 percent benefit from this intervention. Vancomycin is used in more severe cases and in subsequent episodes; combinations of antimicrobials have also been tried in some cases and treatment guidelines and algorithms are available to help in the management of CDI cases [2].

A potential target in the development of drugs for the treatment of $C$. difficile infections is aminoacyl-tRNA synthetase (AaRS). Aminoacyl-tRNA synthetases, can exist as either homodimers or monomers and catalyze the specific aminoacylation of aminoacid transfer RNAs [4]. Class I aminoacylsynthetases consist of our domains: a catalytic Rossmann fold, a connective peptide (CP) inserted between the two halves of the Rossmann fold, a KMSK domain and a C-terminal $\alpha$-helix bundle domain, responsible for the recognition of the CAU anticodon of aminoacyl tRNAs [5], whereas class II synthetases are characterized by a catalytic core of antiparallel $\beta$-sheets and three consensus motifs [6].

Although the natural substrates for AaRS enzymes are the same for all living organisms, the mode of binding of these amino acid substrates can be different depending on several factors including the active site conformation. In recent years increasing numbers of compounds have been reported to inhibit AaRS enzymes, many of them however inhibit human enzymes [7], which can cause toxicological issues. Success in identifying molecules that specifically inhibit 
bacterial AaRS enzymes has recently been achieved [7]. Success in clinical applications started with Mupirocin (marketed as Bactroban), which is the first AaRS inhibitor antibiotic that selectively inhibits bacterial isoleucyl-tRNA synthetase [7, 8]. Mupirocin is now the first choice topical antibiotic for the treatment of methicillin-resistant Staphylococcus aureus (MRS A).

Several potent nanomolar S. aureus methionyl tRNA synthetase (MetRS) inhibitors were developed and found to have excellent antibacterial activity against Staphylococcal and Enterococcal pathogens [9-12]. One of the quinolinone derivatives tested selectively inhibited S. aureus MetRS and was found to be effective in vivo in rat [9]. Very interesting is the generation of REP3123, which is a potent MetRS inhibitor of $C$. difficile [13]. REP3123 was developed as an anti-microbial agent for treatment of $C$. difficile associated diseases (CDAD) and showed potent antimicrobial activity against $C$. difficile and Gram-positive aerobic bacteria; however it does not affect the normal gut flora content. REP3123 prevents $C$. difficile sporulation and therefore inhibits toxin production. The compound is an effective inhibitor of both $C$. difficile and $S$. aureus MetRS and does not inhibit human MetRS [13]. The success of REP3123 as an inhibitor of $C$. difficile MetRS provides a useful ligand, in addition to known substrates of MetRS, to develop and evaluate a homology model.

There are no crystal structures of the AaRS enzymes from $C$. difficile, however comparative structure modeling can be used to construct a putative structure for the desired $C$. difficile MetRS enzyme that may facilitate inhibitor development. The construction and subsequent evaluation of a $C$. difficile MetRS homology model is described.

\section{Methods}

\section{Construction of the $C$. difficile MetRS model}

\section{Homology searching}

The $C$. difficile MetRS sequence was obtained from the ExPASy proteomics server [14]. The enzyme sequence has the Uniprot identifier Q181D9 and was obtained from $C$. difficile strain 630, it has 645 residues [15]. A homology search was performed using NCBI BLAST2 service [16] accessible from the ExPASy server, which was used to align the query against 
sequences in the Protein Data Bank (PDB) [17] and thus the close homologous proteins were identified. The alignment parameters and thresholds used for screening candidate homologues were used with their default values and BLOSUM62 comparison matrix.

The Phylogeny server [18, 19] was used to build a phylogenetic tree for these identified homologous proteins, the query sequence and other MetRS enzymes selected from different organisms.

\section{Multiple sequence and structure alignment}

The secondary structure of $C$. difficile MetRS together with the templates were determined using PSIPRED [20]. The query sequence was then aligned against the most homologues templates, Aquifex aeolicus MetRS, Thermus thermophilus MetRS, Pyrococcus Abyssi MetRS, Escherichia Coli MetRS using Clustal W2 [21] for identification of specific $\alpha$ helices, $\beta$-sheets, coils and loops, and the common features and motifs: 'HIGH region', KMSKS motifs, zinc metal and ATP binding sites. Further evaluation of fold recognition and identification of template structures by structural alignment was performed using mGenTHREADER [22].

\section{D Model Building}

The molecular modeling experiments were performed on a RM Intel Core2 Duo CPU 2.2GHz running Windows XP using Molecular Operating Environment (MOE) 2008.09 molecular modeling software [23]. Homology models were built using MOE-Homology using AMBER99 forcefield [24], which uses a dictionary to set the partial charges of atoms in amino acids. The final homology model was constructed using all the A. aeolicus MetRS (2CSX) crystal structure and the T. thermophilus MetRS zinc finger residues (from 1A8H). MOE-align was used to identify the zinc binding domain residues in the second template ( $T$. thermophilus MetRS). The identified residues were selected to override the main template for building the model, the zinc and the Met-tRNA atoms were selected and included in the model as environment for induced fit, all other setting options were set to the default. Ten intermediate models were generated and the final model was taken as the Cartesian average of all the intermediate models. All the minimizations were performed with MOE until RMSD gradient of $0.05 \mathrm{kcal} \mathrm{mol}^{-1} \AA^{-1}$ with the forcefield specified and the partial charges were automatically calculated. 


\section{Model validation}

Stereochemical quality of the polypeptide backbone and side chains was evaluated using Ramachandran plots obtained from the RAMPAGE server [25]. Amino acid environment was evaluated using ERRAT plots [26]. ERRAT assesses the distribution of different types of atoms with respect to one another in the protein model. The ProSA tool [27] was used to check the overall model protein structure for potential errors. Validation data for the templates A. aeolicus MetRS (2CSX) and T. thermophilus MetRS (1 A8H) was used as the baseline to assess the respective models.

\section{Docking}

Ligand structures were obtained from the relevant complex crystal structures, REP3123 was built using MOE-builder [23] and then the ligand was energy minimized. These ligands were docked using the MOE-Dock with setting: Placement: Triangle Matcher; Rescoring 1: London $\mathrm{dG}, 30$ poses were constructed for each compound and the best scoring model-ligand complexes were selected; the ligand interactions within these complexes were visualized using the MOE ligand interactions simulation.

\section{Results and discussion}

\section{Homology models and validation}

Initial screening for possible templates performed by running a BLAST analysis [16] of the amino acid sequence of $C$. difficile MetRS, obtained from the ExPASy proteomics server [14], against the PDB resolved structures, identified five structures for further consideration as possible templates (Table 1). For a structure to be considered it should ideally be wild-type, rather than mutant or engineered, have reasonable sequence identity with the $C$. difficile MetRS and preferably have the same function. The first ten native hits are bacterial MetRS enzymes, with the MetRS enzyme of Aquifex aeolicus (A. aeolicus) being the most appropriate template due to the high sequence identity (48\%). The Thermus thermophilus (T. thermophilus) enzyme also had a good sequence identity of $39 \%$. In addition Escherichia Coli (E. coli) MetRS (23\% identity) and Pyrococcus Abyssi (P. Abyssi) MetRS (28\% identity) can 
still be considered to have a reasonable sequence similarity. These MetRS enzymes constitute the dataset of suggested templates.

$<$ Table 1>

The MetRS enzymes have common features and motifs: 'HIGH region', KMSKS motif, zinc metal and ATP binding sites (Table 2). Class I AaRS enzymes are characterized by a catalytic center built around a Rossmann fold where the two signature sequences HIGH and KMSKS are found $[5,28]$. The HIGH motif is located in a loop between $\beta 1$ and $\alpha 1$ elements, with the two histidine residues stacked upon each other [28]. The KMSKS peptide in located in a $\beta-\alpha-$ $\alpha-\beta-\alpha$ structure, named the stem contact (SC) fold [29, 30]. The HIGH region has been implicated in amino acid activation and the KMSKS in docking the acceptor stem of tRNA. The positions of these features in different species are varied or shifted by a few residues but are still present in all of them [5].

$<$ Table 2>

To obtain more information regarding the best possible template, the Phylogeny server [18, 19] was used to construct a phylogenetic tree using sixteen more MetRS enzyme sequences in addition to the query and the above four suggested MetRS templates. A diverse range of species was considered including mammals, fish, yeast and bacteria so as to appreciate the relative distances between the suggested four templates and the query sequence (Fig. 1).

$<$ Figure 1>

The enzyme sequences of $S$. aureus and $C$. acetobutylicum were the closest to the query sequence in the phylogenetic tree. However, by considering the next closest branch, it was interesting to observe that the enzyme sequences of $A$. aeolicus and $T$. thermophilus were the next closest. The other two suggested templates of $E$. coli and $P$. Abyssi were diverting in branches relatively far from the query sequence.

\section{Multiple sequence and structural alignments}

ClustalW2 [21] was used to align the suggested template sequences and the query sequence of C. difficile MetRS (Fig. 2). 
$<$ Figure 2>

Very few gaps in alignment were observed when considering the $C$. difficile, $T$. thermophilus and $A$. aeolicus MetRS sequences alone until approximately residue 500. However, consistent gaps were observed between these three sequences in respect to the other two suggested templates of E. coli and Pyrococcus Abyssi MetRS sequences. The difference between the two groups was in agreement with the relations described by the phylogenetic tree (Fig. 1). HIGH and KMSKS motifs are identified in all suggested templates and query sequence (Fig. 2).

To compare structural alignment, the $C$. difficile MetRS protein sequence was used as a template for the mGenTHREADER program [22], to search the Protein DataBank (PDB) for proteins that are likely to contain similar structural motifs. The mGenTHREADER program is a recommended method for fold recognition and identification of distant homologues and makes use of profile-profile alignments and predicted secondary structure (using PSIPRED) as inputs. The GenTHREADER method also runs PSI-BLAST on the target sequence before the search. A. aeolicus (45\% identity) and T. thermophilus (38\% identity) are likely the closest proteins structurally (Table 3 ).

$<$ Table 3>

Template analysis therefore suggested A. aeolicus MetRS as the main template, however the A. aeolicus MetRS crystal structure (2CSX) lacks the zinc binding domain involving residues 124 to 158. Employing the missing sequence folds from T. thermophilus MetRS rectified this problem. This multi-template approach is quite appropriate here because the missing residues are found clustering in the structure just around the zinc. Visualization of both templates in MOE by superimposition has shown that the T. thermophilus $(1 \mathrm{~A} 8 \mathrm{H})$ and A. aeolicus (2CSX) MetRS structures overlap well with the zinc binding domain of $1 \mathrm{~A} 8 \mathrm{H}$ clearly identified (Fig. $3)$.

$<$ Figure $3>$

Both the T. thermophilus and A. aeolicus MetRS sequences are too short to align with the $C$. difficile MetRS sequence $\mathrm{C}$ terminus. The $\mathrm{C}$ terminal structure of $P$. Abyssi MetRS (1MKH) seems to be the closest in terms of sequence identity and secondary structure; however a considerable gap in alignment of the $\mathrm{C}$ terminal sequence would prevent using it as a template 
for modeling the $\mathrm{C}$ terminus alone. It has been observed that many crystal structures of MetRS have an incomplete $\mathrm{C}$ terminal structure. For example, the structure of $P$. Abyssi MetRS (1RQG) lacks the whole C-terminus. The C-terminal domain is less well conserved overall among the MetRS enzymes, which makes modeling this domain challenging [4, 31]. Studies of the C-terminal domains of P. Abyssi MetRS [32] and E. coli MetRS [33] have indicated that the enhancement of tRNA-binding affinity conferred by the C-terminal domain is only expressed when the enzyme is in the dimeric state and, for E. coli MetRS, the enzyme remains active in monomeric form after removal of a $\mathrm{C}$-terminal region required for dimerization [33]. By weighing the risks and assessing the need for modeling the $\mathrm{C}$ terminus of the query, it was decided not to incorporate an unsatisfactory $\mathrm{C}$-terminus crystal structure in the multiple template combination, which may adversely affect the rest of the model.

PSIPRED [20] secondary structure prediction from the mGenTHREADER program [22] for C. difficile MetRS clearly showed the high helix content predicted throughout the sequence. The C-terminus had a higher degree of coils and strands predicted than for the rest of the protein sequence, this in addition to the poor alignment observed for the C-terminus would make the $\mathrm{C}$ terminus a challenging sequence to model. The HIGH region motif (Table 2) is a sequence of about ten residues and was found in most of the homologous enzymes at the sequence positions just before the $22^{\text {nd }}$ residue in the $\mathrm{N}$ termini of these enzymes. This particular area of the query sequence was expected by PSIPRED to fold in coils and strands, this holds for A. aeolicus where the resolved structure shows residues 6-15 form a strand very close to where the HIGH region motif was suggested. The KMSKS motif is another known motif among the MetRS enzymes described. The motif contains the ATP binding site and has been found in the query sequence aligned to the $T$. thermophilus and $A$. aeolicus sequences with less than a three position differences in both cases (Table 2). The A. aeolicus 3D structure contains strands and turns from position 283 until at least 301 . This is very much in agreement with the predictions of PSIPRED for the query sequence.

\section{Zinc binding}

Zinc is an absolute requirement for enzyme activity [34]. There are two steps associated with the enzyme activity catalyzing the transfer of the Met amino acid into the cognate tRNA: tRNAMet aminoacylation and ATP-pyrophosphate exchange activities. It is certain now that the zinc should be in the actual structure of the enzyme and that it is most likely bound to four 
cysteine residues in the query protein and hence the intended query model. This was supported by the alignment of $C$. difficile MetRS with the sugges ted templates where several conserved Cys residues and a histidine residue (E. coli) are identified in the likely region of this domain in the query sequence (Fig. 2).

\section{D Homology model}

Homology models, constructed using all the A. aeolicus MetRS (2CSX) crystal structure and the $T$. thermophilus MetRS zinc finger residues $(1 \mathrm{~A} 8 \mathrm{H})$, were generated using Molecular Operating Environment (MOE) [23] software as described in the experimental section. Using combined templates to generate the $C$. difficile MetRS model allowed identification of the cysteine residues binding the zinc ion. These cysteine residues were all near the position of the zinc, at least three of which had the sulfur directly pointing towards the zinc. For the fourth cysteine residue side chain manipulation using MOE-rotamer explorer was employed to improve the zinc binding. The sulfur atoms binding the zinc at the constructed model were from Cys128, Cys131, Cys146 and Cys149 (Fig. 4), also noted in close proximity was Asp148 that could also chelate with the zinc ion.

$<$ Figure 4>

The Met tRNA sequence of $C$. difficile, available from the National Centre for Biotechnology Information [34, 35] consists of 73 bases, similar to that of A. aeolicus. Moreover, the CAU anticodon positions are also the same. RNAfold [36], a web server for predicting the RNA secondary structure, indicated that the A. aeolicus Met tRNA secondary structure is quite similar to the predicted C. difficile Met tRNA structure using the RNA fold (Fig. 5) therefore the tRNA chain of $A$. aeolicus MetRS was used in the query MetRS homology modeling.

\section{$<$ Figure 5>}

Asn356, Arg360 and Trp430 of C. difficile MetRS are fully involved in binding the anticodon part of Met-tRNA (Fig. 6). The corresponding residues in the MetRS enzymes of A. aeolicus, T. thermophilus and E. coli play the same role. Asn356 and Arg360 form hydrogen bonds with 35A and 34C. As seen in the A. aeolicus MetRS complex the Trp residue (422 in A. aeolicus, Trp430 in the query sequence) is not involved in direct interaction with the anticodon $36 \mathrm{U}$ but interacts by hydrogen bond with a Phe residue (492 in A. aeolicus and 
Phe508 in the query sequence). While these findings are predictable by literature mining along with sequence alignments, having found that the model met these expectations can give some confidence to additional observations: Lys367 and Lys434 of C. difficile MetRS interact with 35 A and 34C while Leu507 and Arg510 interact with 36U (Fig. 7).

$<$ Figure 6>

The final C. difficile MetRS model (Fig. 7A) contained 513 residues from the 645 residues of the sequence; this was the result of disabling the $\mathrm{C}$-terminus. The secondary structure of these residues involved twenty $\alpha$-helices and $14 \beta$-sheets (Table 4). This model also contained a zinc metal and A. aeolicus tRNA.

$<$ Figure 7>

$<$ Table 4>

The Rossmann fold domain is formed by two polypeptide segments (residues 1-115 and 229289, colored orange in Fig. 7B). Consistent with other AaRS enzymes [29, 37, 38], the last residue of the Rossmann fold, His 289 in the $\beta 12$ strand, forms a hydrogen bond the backbone $\mathrm{N}-\mathrm{H}$ group of Val259 in the $\beta 11$ strand and is followed by a glycine residue. The HIGH motif of $C$. difficile MetRS (His20-Ile21-Gly22-His23) is located between $\beta 2$ and $\alpha 1$, with the two histidine residues stacked upon each other.

Inserted between the two Rossmann fold polypeptide segments is the connective polypeptide (CP) domain, composed of an antiparallel $\beta$ sheet core (cyan color, Fig. 7B), a MetRSspecific $\beta$-strand insertion (green, Fig. 7B), zinc finger (yellow, Fig. 7B) and antiparallel $\alpha_{2}-$ helical structure (blue, Fig. 7B). The $\mathrm{CP}$ core consists of $\beta 5, \beta 6, \beta 7, \beta 8$ and $\beta 9$, between the second and third strands ( $\beta 6$ and $\beta 7$ ) is the zinc finger with one $\mathrm{Zn}^{2+}$ ion coordinated by sulfur atoms (Cys128, Cys131, Cys146 and Cys149, Fig. 4). In T. thermophilus MetRS the zinc bind ing domain consists of an antiparallel $\beta$-sheet insertion involving $3 \beta$-strands [29]. In this C. difficile MetRS model, the zinc binding domain consists of a loop between $\beta 5$ and $\beta 6$ with an $\alpha$-helix $(\alpha 5)$ positioned in the middle of the four zinc-coordinating cysteine residues. Between the second and third $\beta$-strands ( $\beta 6$ and $\beta 7$ ), two antiparallel $\alpha$-helices ( $\alpha 6$ and $\alpha 7$ ) are inserted (Fig. 7B), and between the last antiparallel $\beta$-sheet domain and the C-terminal 
Rossmann fold domain, a $\beta$-strand ( $\beta 10$, Val225-Ile224, green in Fig. 7B) is inserted in an antiparallel manner. This $\beta$-strand insertion is considered to be a MetRS specific element [29].

The region connecting the $\mathrm{N}$-terminal Rossmann fold (orange, Fig. 7B) with the C-terminal $\alpha$-helix bundle domain (magenta, Fig. 7B) is the SC-fold domain (red, Fig. 7B). The SC-fold domain, which is the RNA binding domain, has a $\beta-\alpha-\alpha-\beta-\alpha(\beta 13-\alpha 12-\alpha 13-\beta 14-\alpha 14)$ topology. This homologous topology is well conserved and characteristic of the monomeric class Ia and Ib synthetase enzymes [29, 37, 38]. The KMSKS (Lys298-Met299-Ser300Lys291-Ser292) motif is situated in the SC-fold domain in a loop between $\beta 13$ and $\alpha 12$.

The anticodon binding $\alpha$-helix bundle domain (magenta, Fig. 7B) consists of five antiparallel $\alpha$-helices $(\alpha 15-\alpha 19)$. The amino acid residues involved in anticodon recognition are clustered on one face of the $\alpha$-helix bundle domain, with Asn356, Arg360 and Lys367 in the $\alpha 14$ helix and Trp430 and Lys434 in the $\alpha 16$ helix.

\section{Model validation}

To evaluate the quality of the modeled structures, the lowest energy model generated was subjected to a number of checks. Stereochemical quality was assessed us ing Ramachandran plots, using the Cambridge RAMPAGE server [25], and amino acid environment was assessed using ERRAT [26]. Overall protein structure was assessed using ProS A [27].

Validation results would suggest that the model performed well in terms of mainchain stereochemistry and amino acid environment. In the Ramachandran plot, a total of $97.9 \%$ of the residues were in the allowed region, which was comparable with the main template 2CSX (97.1\%), indicating that the backbone dihedral angles $\psi$ and $\phi$ in the model were reasonably accurate. There were 11 outliers in the model however they were far from the active site and do not significantly contribute to its function. The ERRAT results showed that the overall quality factor of the protein was good with a score of 86.139 compared with the main template 2CSX (84.862). It is surprising for the model to perform better than the template, however the template was missing the $\mathrm{Zn}$-binding domain residues. The $\mathrm{Zn}$-binding template 1A8H scored better in both Ramachandran $(99.4 \%$ of residues in the allowed region, 3 outliers) and ERRAT (98.374) evaluation, therefore the combined template approach has 
incorporated some improvements in stereochemical quality compared with the 2CSX template alone.

$<$ Figure 8>

ProSA analysis provides output in two plots: the first plot (Fig. 8A) shows the local model quality by plotting energies as a function of amino acid sequence position, in general, positive values correspond to problematic or erroneous parts of the input structure. The second plot (Fig. 8B) shows overall model quality from which the z-score is calculated, its value is displayed in a plot that contains the z-scores of all experimentally determined protein chains in the current PDB, a negative score indicates a good model whereas a positive score would indicate errors. The $\mathrm{z}$-score of the model was -10.57 compared with 2CSX (-11.79) and 1A8H (-12.72). Furthermore, superimposition of the model with the main template 2CSX showed a very low RMSD (0.816 ̊) suggesting a high similarity between them, superimposition with $1 \mathrm{~A} 8 \mathrm{H}$ resulted in a higher RMSD (2.198 $)$, however as only the zinc finger residues were used from $1 \mathrm{~A} 8 \mathrm{H}$ this was not expected to have a detrimental effect on the model.

Overall based on Ramachandran plot, ERRAT and ProS A a good model, in terms of quality of the local geometry of the backbone and side-chain conformations, for $C$. difficile MetRS was generated. Further validation of the active site architecture and key binding interaction were achieved by substrate and ligand docking experiments.

\section{Active site analysis and docking}

By using the ClustalW2 multiple sequence alignment [21] and the MOE alignment service [23], the positions of the active site conserved residues in the query sequence, A. aeolicus, E. coli and T. thermophilus MetRS can be identified. Docking suitable ligands into the constructed model was used to validate the predicted active site. The docked ligands include the MetRS natural substrate (methionine), several E. coli MetRS inhibitors 5'-O-[N-(Lmethionyl)-sulfamoyl] (MSP), difluoromethionine (2FM) and trifluoromethionine (MF3), (1amino-3-methylsulfanyl-propyl)-phosphonic acid (MPH) and a C. difficile MetRS inhibitor (REP3123), no site restriction was specified in the MOE docking procedures. 
Docking the natural substrate, methionine, identified key residues with His53, Asp51 and Lys56 consistently forming H-bonding interactions with the carboxylic acid moiety of methionine. Ile12 was also found to hydrogen bond with this moiety in different dockings and the residues Ile224, Va1226 and Ala230 forming a hydrophobic pocket (Table 5).

\section{$<$ Table 5>}

The MSP ligand was docked in the $C$. difficile MetRS model. It was observed that the residues around the docked ligand remained almost the same in all the dockings (Table 5). The following residues interact with the ligand in different docking attempts: Ile12, His20, Asp51, Lys56, Val226, Trp227 and Trp291 with H-bonding interactions most frequently observed with Asp51, Lys56 and Ile12. Difluoromethionine (2FM) and trifluoromethionine (MF3) are only available bound to the E. coli MetRS [39]. MOE dockings of MF3 showed that Asp51 of the C. difficile MetRS model is consistently interacting with the ligand. Ile12, Asp51, His267, Val226, Trp227, Leu233 and Ile263 are in the model binding pocket surrounding the ligand and are very close to the expectations derived from E. coli MetRS complex with MF3 (Table 5).

2FM was found to dock in two sites within the active site, the first was the site involving interaction with Asp51 and Lys56 seen previously with methionine, MSP and MF3 and the second site involved interaction with Glu55, Ser133 and Tyr225. Many conserved residues in the $\mathrm{N}$ terminal and in positions from 224 to 240 and from 255 to 268 are also found in the 2FM dockings (Table 5). Docking of 1-amino-3-methylsulfanyl-propyl)-phosphonic acid (MPH), found in the resolved complex structure of $E$. coli MetRS (1P7P) [40] resulted in diverse ligand interactions but comparable with those found when docking $2 \mathrm{FM}$, that is MPH docked in two distinct binding sites within the active site. Again the first was the site involving interaction with Asp51 and Lys56 seen previously with MSP and MF3 and the second site involved interaction with Glu55, Ser133 and Tyr225 (Table 5).

The binding mode of ligands in the $C$. difficile MetRS model was comparable with the binding of identical ligands in E.coli crystal structures (PDB identifier: 1F4L (methionine); 1PFY (MSP); 1PFV (2FM); 1PFW (MF3); 1P7P (MPH). In these crystal structures the key $\mathrm{H}$-bonding interactions involved the conserved residues Leu13 and Asp52, with the residues Ala256 (Ala230 in C.difficile), Pro257, Tyr260, Ile297 (Ile263 in C.difficile) and His301 (His267 in $C$. difficile) forming hydrophobic interactions. The overlap in binding residues 
between identical ligands and the $C$. difficile MetRS model and E. coli MetRS crystal structures demonstrates that the modeled structure is of sufficient quality to correctly reproduce ligand binding poses in docking simulations.

REP 3123 is the only ligand found in the literature that has been described as a selective $C$. difficile MetRS inhibitor [13]. The antimicrobial effect is described and the chemical structure of the inhibitor. It was confirmed in their study that REP 3123 is a selective and potent competitive inhibitor of $C$. difficile MetRS, which implies that the ligand has features that enable it to strongly bind at the active site of $C$. difficile MetRS. REP 3123 is not a competitive inhibitor of ATP and the presence of ATP facilitated inhibition [13], therefore it could be deduced that the ligand binds by a similar mechanism as methionine. REP 3123 does not inhibit E. coli MetRS however it does inhibit S. aureus MetRS [13], which may be explained by the close position of $C$. difficile and $S$. aureus MetRS indicated by the phylogenic tree (Fig. 1).

$<$ Figure 9>

Docking of REP3123 indicated that this ligand docks across both binding sites, the first pocket is formed by Asp51, Ile12, Ala230 and Trp227 and the second pocket, linked by Lys56 and Tyr14, is formed by Glu55, Ser133, Tyr225 and Gln139 (Fig. 9). Interestingly a pharmacophore virtual screening study by Finn et al [10], employed to develop S. aureus MetRS inhibitors, identified Asp51 as a key residue with optimal inhibitory activity observed on formation of two hydrogen bond interactions between the ligand and the critical Asp51 group. Another study investigating the effect of resistance mutations on S. aureus MetRS activity found that Gly54 (G54S) and Gly223 (G223C) mutations were the most deleterious single mutants with markedly impaired enzymatic function [41]. The high sequence identity (50\%) between $S$. aureus MetRS and $C$. difficile MetRS may suggest that the proximity of Glu55 to Gly54 in the binding pocket would indicate the importance of Glu55 in binding interactions and inhibition of enzyme function. The ability of REP3123 to interact with both binding pockets and form H-bonding interactions with several residues including Asp51, Ile12, Lys56, Glu55, Ser133 and Tyr225 may account for the high selectivity and inhibitory activity against $C$. difficile MetRS. 


\section{Conclusions}

Development of a homology model for MetRS has been explored using a multi-template approach and the generated C.difficile MetRS model has the characteristic domains and topology found in class I AaRS enzymes. Docking suitable ligands into the constructed model was then used to validate the predicted active site. The docked ligands included the MetRS natural substrate (methionine), several E. coli MetRS inhibitors and a C. difficile MetRS inhibitor (REP3123), no site restriction was specified in the MOE docking procedures. Both conservation analysis and docking studies have agreed to the same active site with two pockets identified, the active site key residues in the model, which span the C-terminal Rossmann fold and the CP domain, include Ile12, Asp51, Ala230, Trp227 (pocket 1), Glu55, Ser133 and Tyr225 (pocket 2) with Lys56 bridging the two pockets. The ability to interact with both pockets within the binding site with multiple hydrogen bonding interactions, as observed for REP3123, may be advantageous for inhibitory activity. The Met-tRNA binding within the constructed model has also been validated. In the constructed model, Asn356, Arg360 and Trp430 were conserved and involved in the specific interactions between the protein and the Met-tRNA anticodon (CAU), the corresponding residues in close homologous MetRS have the same binding role. Having identified both the active site pockets of $C$. difficile MetRS and key binding interactions, this model can serve as a tool for drug design and the development of inhibitors with greater selectivity can be explored. 


\section{References}

1. Richmond MA, Riggs MM, Eckstein BC, Donskey CJ (2008) Clostridium difficile infection in patients with SCI. J Spinal Cord Med 31:521-521

2. Gerding DN, Muto CA, Owens RC (2008) Treatment of Clostridium difficile infection. Clin Infect Dis 15:S32-42

3. Deaths involving Clostridium difficile: England and Wales 2004-2008 Office for National Statistics 19/8/2009. http://www.statistics.gov.uk

4. Deniziak MA, Barciszewski J (2001) Methionyl-tRNA synthetase. Acta Biochim Pol 48:337-350

5. Hountondji C, Dessen P, Blanquet S (1986) Sequence similarities among the family of aminoacyl-tRNA synthetases. Biochimie 68:1071-1078

6. Eriani G, Delarue M, Poch O, Gangloff J, Moras D (1990) Partition of transfer-RNA synthetases into 2 classes based on mutually exclusive sets of sequence motifs. Nature 347:203-206

7. Hurdle JG, O'Neill AJ, Chopra I (2005) Prospects for aminoacyl-tRNA synthetase inhibitors as new antimicrobial agents. Antimicrob Ag Chemother 49:4821-4833

8. Boyce JM (2001) MRSA patients: proven methods to treat colonization and infection. J Hosp Infect 48: S9-14

9. Jarvest RL, Berge JM, Berry V, Boyd HF, Brown MJ, Elder JS, Forrest AK, Fosberry AP, Gentry DR, Hibbs MJ, Jaworski DD, O'Hanlon PJ, Pope AJ, Rittenhouse S, Sheppard RJ, Slater-Radosti C, Worby A (2002) Nanomolar inhibitors of Staphylococcus aureus methionyl tRNA synthetase with potent antibacterial activity against gram-positive pathogens. J Med Chem 45:1959-1962

10. Finn J, Mattia K, Morytko M, Ram S, Yang Y, Wu X, Silverman J, Mak E, Gallant P, Keith D (2003) Discovery of a potent and selective series of pyrazole bacterial methionyl-tRNA synthetase inhibitors. Bioorg Med Chem Lett 13:2231-2234

11. Tandon M, Coffen D, Gallant P, Keith D, Ashwell M (2004) Potent and selective inhibitors of bacterial methionyl tRNA synthetase derived from an oxazolone-dipeptide scaffold. Bioorg Med Chem Lett 14:1909-1911

12. Finn J, Stidham M, Hilgers M, Kedar GC (2008) Identification of novel inhibitors of methionyl-tRNA synthetase (MetRS) by virtual screening. Bioorg Med Chem Lett $18: 3932-3937$ 
13. Critchley IA, Green LS, Young CL, Bullard JM, Evans RJ, Price M, Jarvis TC, Guilles JW, Janjic N, Ochsner U (2009) Spectrum of activity and mod of action of REP3123, a new antibiotic to treat Clostridium difficile. Antimicrob Ag Chemother 63:954-963

14. Gasteiger E, Gattiker A, Hoogland C, Ivanyi I, Appel RD, Bairoch A (2003) ExPASy: the proteomics server for in-depth protein knowledge and analysis. Nucleic Acids Res 31:3784-3788. www.expasy.org

15. Sebaihia M, Wren BW, Mullany P, Fairweather NF, Minton N, Stabler R, Thomson NR, Roberts AP, Cerdeño-Tárraga AM, Wang H, Holden MT, Wright A, Churcher C, Quail MA, Baker S, Bason N, Brooks K, Chillingworth T, Cronin A, Davis P, Dowd L, Fraser A, Feltwell T, Hance Z, Holroyd S, Jagels K, Moule S, Mungall K, Price C, Rabbinowitsch E, Sharp S, Simmonds M, Stevens K, Unwin L, Whithead S, Dupuy B, Dougan G, Barrell B, Parkhill J (2006) The multidrug-resistant human pathogen Clostridium difficile has a highly mobile, mosaic genome. Nat Genet 38:779-786

16. Altschul SF, Madden TL, Schäffer AA, Zhang J, Zhang Z, Miller W, Lipman DJ (1997) Gapped BLAST and PSI-BLAST: a new generation of protein database search programs. Nucleic Acids Res 25:3389-3402

17. RCSB Protein Data Bank (PDB) http://www.rcsb.org/pdb

18. http://www.phylogeny.fr/

19. Dereeper A, Guignon V, Blanc G, Audic S, Buffet S, Chevenet F, Dufayard JF, Guindon S, Lefort V, Lescot M, Claverie JM, Gascuel O (2008) Phylogeny.fr: robust phylogenetic analysis for the non-specialist. Nucleic Acids Res 36:W465-469

20. Jones DT (1999) Protein secondary structure prediction based on position-specific scoring matrices. J Mol Biol 292:195-202

21. Larkin MA, Blackshields G, Brown NP, Chenna R, McGettigan PA, McWilliam H, Valentin F, Wallace IM, Wilm A, Lopez R, Thompson JD, Gibson TJ, Higgins DG (2007) ClustalW and ClustalX version 2. Bioinformatics 23:2947-2948

22. Lobley A, Sadowski MI, Jones DT (2009) pGenTHREADER and pDomTHREADER: New Methods For Improved Protein Fold Recognition and Superfamily Discrimination. Bioinformatics $25: 1761-1767$

23. Molecular Operating Environment (MOE 2008.09) Chemical Computing Group Inc, Montreal Quebec Canada http://www.chemcomp.com. 2008.09

24. Weiner SJ, Kollman PA, Nguyen DT (1986) An all atom forcefield for simulations of proteins and nucleic acids. J Comput Chem 7:230-252

25. RAMPAGE Server http://ravenbioccam.ac.uk/rampage.php 
26. UCLA-DOE Institute for Genomics \& Proteomics Server http://www.doembiucla.edu/Services

27. Wiederstein M, Sippl MJ (2007) ProSA-web: interactive web service for the recognition of errors in three-dimensional structures of proteins. Nucleic Acids Res 35:W407-W410

28. Landes C, Perona JJ, Brunie S, Rould MA, Zelwar C, Steitz TA, Risler JL (1995) A structure-based multiple sequence alignment of all class I aminoacyl-tRNA synthetases. Biochemie 77:194-203

29. Sugiura I, Nureki O, Ugaji-Yoshikawa Y, Kuwabara S, Shimada A, Tateno M, Lorber B, Giege R, Moras D, Yokoyama S, Konno M (2000) Structure 8 :197-208

30. Kamijo S, Fujii A, Onodera K, Wakabayashi K (2009) Analysis of conditions for KMSS loop in Tyrosyl-tRNA synthetase by building a mutant library. J Biochem 146:241-250

31. Spencer AC, Heck A, Takeuchi N, Watanabe K, Spremulli LL (2004) Characterization of the human mitochondrial methionyl-tRNA synthetase. Biochemistry 43:9743-9754

32. Crepin T, Schmitt E, Blanquet S, Mechulam Y (2004) Three-dimensional structure of methionyl-tRNA synthetase from Pyrococcus abyssi. Biochemistry 3:2635-2644

33. Mechulam Y, Schmitt E, Maveyraud L, Zelwer C, Nureki O, Yokoyama S, Konno M, Blanquet S. (1999) Crystal structure of Escherichia coli methionyl-tRNA synthetase highlights species-specific features. J Mol Biol 294:1287-1297

34. Xu B, Krudy GA, Rosevear PR (1993) Identification of the metal ligands and characterization of a putative zinc finger in methionyl-tRNA synthetase. J Biol Chem 268:16259-16264

35. RNAfold server: http://rna.tbi.univie.ac.at/cgi-bin/RNAfold.cgi

36. Mathews DH, Sabina J, Zucker M, Turner H (1999) Expanded sequence dependence of thermodynamic parameters provides robust prediction of RNA secondary structure. J Mol Biol 288:911-940

37. Cavarelli J, Delagoute B, Eriani G, Gangloff J, Moras D (1998) L-arginine recognition by yeast arginyl-tRNA synthetase. EMBO J 17:5438-5448

38. Nureki O, Vassylyev DG, Katayanagi K, Shimizu T, Sekine S, Kigawa T, Miyazawa T, Yokoyama S, Morikawa K (1995) Architectures of class-defining and specific domains of glutamyl-tRNA synthetases. Science 267:1958-1965

39. Morales AJ, Swairjo MA, Schimmel P (1999) Structure-specific tRNA-binding protein from the extreme thermophile Aquifex aeolicus. EMBO J 18:3475-3483

40.Crepin T, Schmitt E, Mechulam Y, Sampson PB, Vaughan MD, Honek JF, Blanquet S (2003) Use of analogues of methionine and methionyl adenylate to sample 
conformational changes during catalysis in Escherichia coli methionyl-tRNA synthetase. J Mol Biol 332:59-72

41. Green LS, Bullard JM, Ribble W, Dean F, Ayers DF, Ochsner UA, Janjic N, Jarvis TC (2009) Inhibition of methionyl-tRNA synthetase by REP8839 and effects of resistance mutations on enzyme activity. Antimicrob Ag Chemother 53:86-94 


\section{Tables}

Table 1 The five optimal crystal templates identified in the $C$. difficile MetRS BLAST results

\begin{tabular}{|l|l|l|l|l|l|l|}
\hline Enzyme in Organism & PDB & Blast & Sequence & Seq. & Chain & E. \\
& code & score & Identity & Identity $\%$ & length & Value \\
\hline Aquifex aeolicus & 2CSX-A & 449 & $232 / 480$ & $48 \%$ & 479 & $\mathrm{e}-127$ \\
\hline Thermus thermophilus & IA8H-A & 363 & $201 / 509$ & $39 \%$ & 500 & $\mathrm{e}-101$ \\
\hline Pyrococcus Abyssi & 1RQG-A & 290 & $206 / 719$ & $28 \%$ & 722 & $7 \mathrm{e}-79$ \\
\hline Escherichia Coli & 1F4L-A & 188 & $131 / 553$ & $23 \%$ & 551 & $5 \mathrm{e}-48$ \\
\hline C terminal of & 1MKH-A & 100 & $50 / 92$ & $54 \%$ & 107 & $1 \mathrm{e}-21$ \\
\hline Pyrococcus Abyssi & & & & & & \\
\hline
\end{tabular}

All were MetRS enzy mes

${ }^{\mathrm{a}}$ The BLAST score for an align ment is calcu lated by summing the scores for each aligned position and the scores for gaps

${ }^{\mathrm{b}}$ (Number of identical residues)/ (length of sequence fragment identified by BLAST) 
Table 2 Key residues of the suggested templates

\begin{tabular}{|l|l|l|l|l|}
\hline PDB & HIGH & KMSKS & Zinc Binding Residues & ATP binding \\
& region motif & motif & & \\
\hline 2CSX-A & $14-18$ & $295-299$ & $129,132,147,150$ & 298 \\
\hline 1A8H-A & $12-22$ & $297-301$ & $127,130,144,147$ & 300 \\
\hline 1RQG-A & $11-21$ & $344-348$ & $143,146,156,159$ & 347 \\
\hline 1F4L-A & $15-25$ & $333-337$ & $146,149,159,162$ & 336 \\
\hline
\end{tabular}


Table 3 mGenTHREADER results for C.difficile MetRS query

\begin{tabular}{|l|l|l|l|l|l|l|l|l|l|l|l|}
\hline Conf & $\begin{array}{l}\text { Net } \\
\text { Score }\end{array}$ & p-Value & $\boldsymbol{E}_{\text {pair }}$ & $\mathbf{E}_{\text {Solv }}$ & $\begin{array}{l}\text { Aln } \\
\text { Score }\end{array}$ & $\begin{array}{l}\text { Aln } \\
\text { Len }\end{array}$ & $\begin{array}{l}\text { Str } \\
\text { Len }\end{array}$ & $\begin{array}{l}\text { Seq } \\
\text { Len }\end{array}$ & $\begin{array}{l}\text { o } \\
\text { Iden }\end{array}$ & $\begin{array}{l}\text { PDB } \\
\text { code }\end{array}$ & Description \\
\hline CERT & 178.758 & $5 e-17$ & -650.4 & -36.9 & 853.3 & 462 & 465 & 645 & 45.2 & 2ct8A0 & Aquifex aeolicus MetRS \\
\hline CERT & 174.608 & $1 e-16$ & -578.6 & -27.4 & 857.0 & 504 & 544 & 645 & 21.0 & 3h9cA0 & Escherichia coli MetRS \\
\hline CERT & 172.541 & $2 e-16$ & -447.3 & -26.9 & 867.0 & 545 & 606 & 645 & 22.4 & 1rqgA0 & Pyrococcus abyssi MetRS \\
\hline CERT & 171.741 & $2 e-16$ & -518.2 & -39.1 & 825.0 & 490 & 500 & 645 & 37.8 & $2 d 5$ bA0 & Thermus thermophilus MetRS \\
\hline CERT & 135.154 & $1 e-12$ & -419.6 & -14.0 & 642.0 & 561 & 862 & 645 & 17.7 & 1 gaxA0 & Thermus thermophilus ValRS \\
\hline CERT & 106.519 & $9 e-10$ & -322.7 & -15.3 & 469.0 & 521 & 821 & 645 & 14.0 & 1ileA0 & Thermus thermophilus IleRS \\
\hline CERT & 103.727 & $2 e-09$ & -256.6 & -6.5 & 476.0 & 580 & 917 & 645 & 13.2 & 1ffyA0 & Staphylococcus aureus IleRS \\
\hline CERT & 97.544 & $7 e-09$ & 535.5 & 13.1 & 389.0 & 362 & 399 & 645 & 9.8 & 3c8zA0 & Mycobacterium smegmatis CysRS \\
\hline CERT & 91.009 & $3 e-08$ & -151.6 & 3.2 & 457.0 & 444 & 628 & 645 & 9.6 & 2zueA0 & Pyrococcus horikoshii ArgRS \\
\hline CERT & 90.077 & $4 e-08$ & -318.0 & -3.9 & 396.0 & 440 & 810 & 645 & 10.9 & 2v0cA0 & Thermus thermophilus LeuRS \\
\hline
\end{tabular}

Conf = Description of confidence level, CERT: $p$-value $<0.0001 ;$ HIGH: $p$-value $<0.001$

Net Score = Raw score from SVM; p-Value = Probability of false positive; PairE = Pairwise energy for model; SolvE = Solvation energy for model; Aln Score $=$ Sequence alignment score; Aln Len = Length of alignment; Str Len = Length of PDB entry; Seq Len = Length of target sequence; $\%$ ID = percentage identical residues between target protein and PDB protein; PDB_ID = PDB identifier (+ chain code + domain code in CATH format) 
Table 4 Secondary structure of C.difficile MetRS model

\begin{tabular}{|c|c|c|}
\hline Residues & $2^{\circ}$ structure & Do main \\
\hline PHE6-TYR9 & $\beta 1$ & \\
\hline ILE12-TYR13 & $\beta 2$ & \\
\hline ILE21-ARG38 & $\alpha 1$ & \\
\hline VAL44-THR50 & $\beta 3$ & Rossmann fold \\
\hline GLU55-ILE62 & $\alpha 2$ & \\
\hline GLU68-MET 86 & $\alpha 3$ & \\
\hline ASP92-A RG95 & $\beta 4$ & \\
\hline LYS99-GLN115 & $\alpha 4$ & \\
\hline 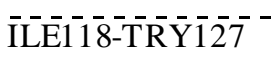 & $\bar{\beta} \overline{5}^{-\cdots}$ & Ántiparallei $\beta$-sheet \\
\hline GLU137-GLN139 & $\alpha 5$ & Zinc-finger \\
\hline LEU155-PHE163 & $\beta 6$ & Antiparallel $\beta$-sheet \\
\hline GLU169-LYS 177 & $\alpha 6$ & $\alpha_{2}$ helical structure \\
\hline ARG187-LYS198 & $\alpha 7$ & $>\mathrm{CP}$ \\
\hline LEU203-SER204 & $\beta 7$ & \\
\hline THR206-A RG207 & $\beta 8$ & Antiparallel $\beta$-sheet \\
\hline LYS215-VAL216 & $\beta 9$ & \\
\hline$\overline{\mathrm{VA}} \overline{\mathrm{L}} \overline{2} \overline{2} \overline{\mathrm{I}}-\overline{\mathrm{L}} \overline{\mathrm{L}} \overline{2} \overline{2} \overline{4}-$ & $\bar{\beta} 10$ & MetRS-specific insertion \\
\hline$\overline{\mathrm{A}} \overline{\mathrm{S} P \overline{2}} \overline{2} \overline{2} \overline{\mathrm{T}}-\overline{\mathrm{T}} \mathrm{HR} \overline{2} \overline{3} \overline{6}^{-}$ & $\alpha 8^{-}$ & - \\
\hline GLU246-LYS250 & $\alpha 9$ & \\
\hline GLN257-GLY260 & $\beta 11$ & \\
\hline LYS261-VAL264 & $\alpha 10$ & Rossmann fold \\
\hline ILE270-A LA277 & $\alpha 11$ & \\
\hline VAL286-HIS 289 & $\beta 12$ & \\
\hline 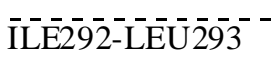 & $\bar{\beta} \overline{1} \overline{-}$ & 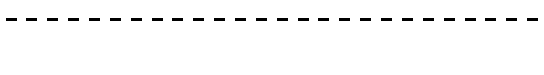 \\
\hline PRO309-ARG315 & $\alpha 12$ & \\
\hline THR320-LEU326 & $\alpha 13$ & Stem contact (SC) fold \\
\hline GLY335-SER336 & $\beta 14$ & \\
\hline HIS339-TYR268 & $\alpha 14$ & \\
\hline$\overline{\mathrm{L}} \overline{\mathrm{EU}} \overline{3} \overline{8} \overline{\mathrm{C}}-\overline{\mathrm{ASP}} 40 \overline{2}$ & $\alpha \overline{15})$ & - \\
\hline PHE406-TYR423 & $\alpha 16$ & \\
\hline PRO429-LYS434 & $\alpha 17$ & $\alpha$-Helix bundle \\
\hline ASP443-ILE460 & $\alpha 18$ & \\
\hline ASN465-ASP481 & $\alpha 19$ & \\
\hline
\end{tabular}


Table 5 Representative dockings into $C$. difficile MetRS model

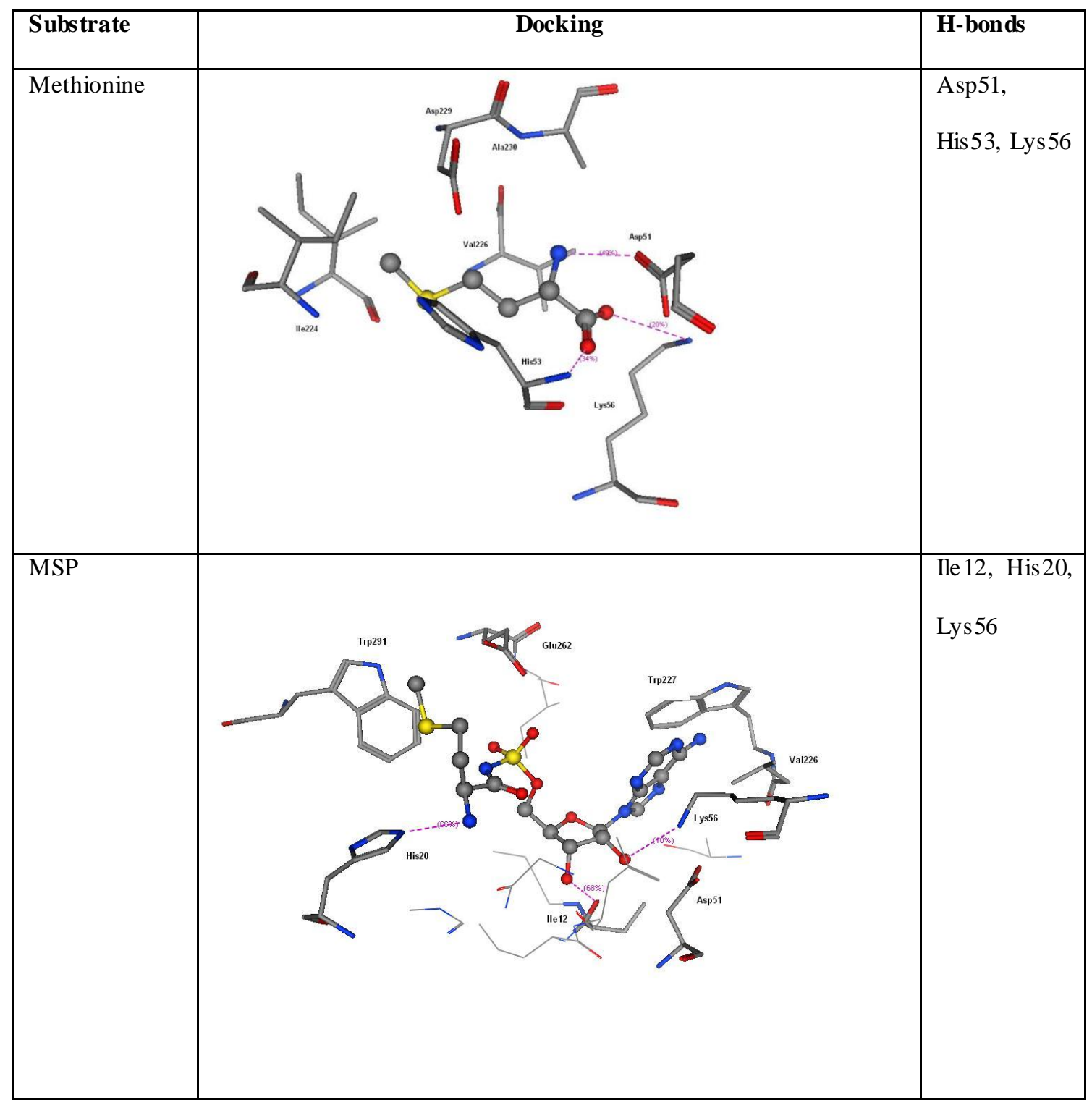




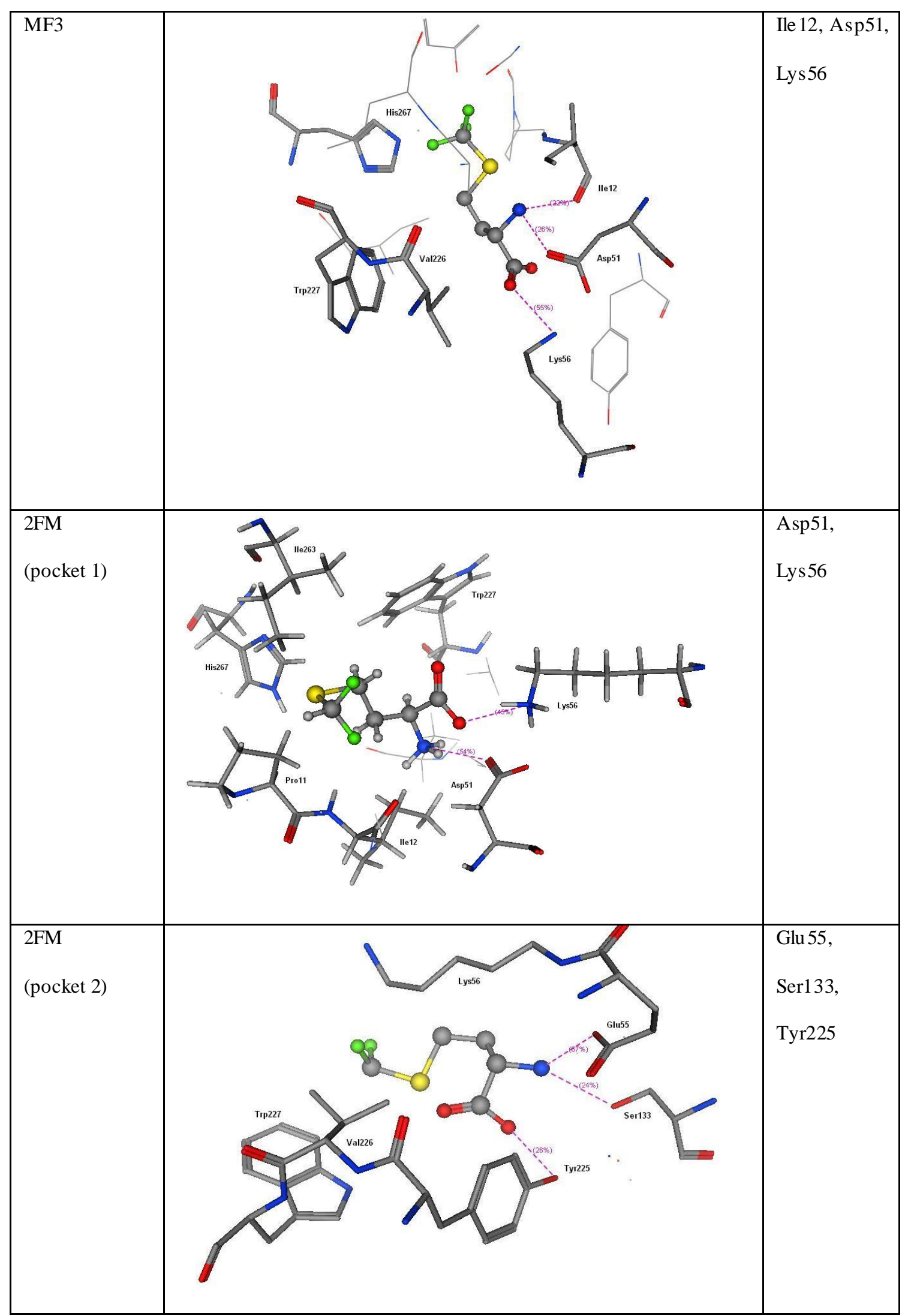




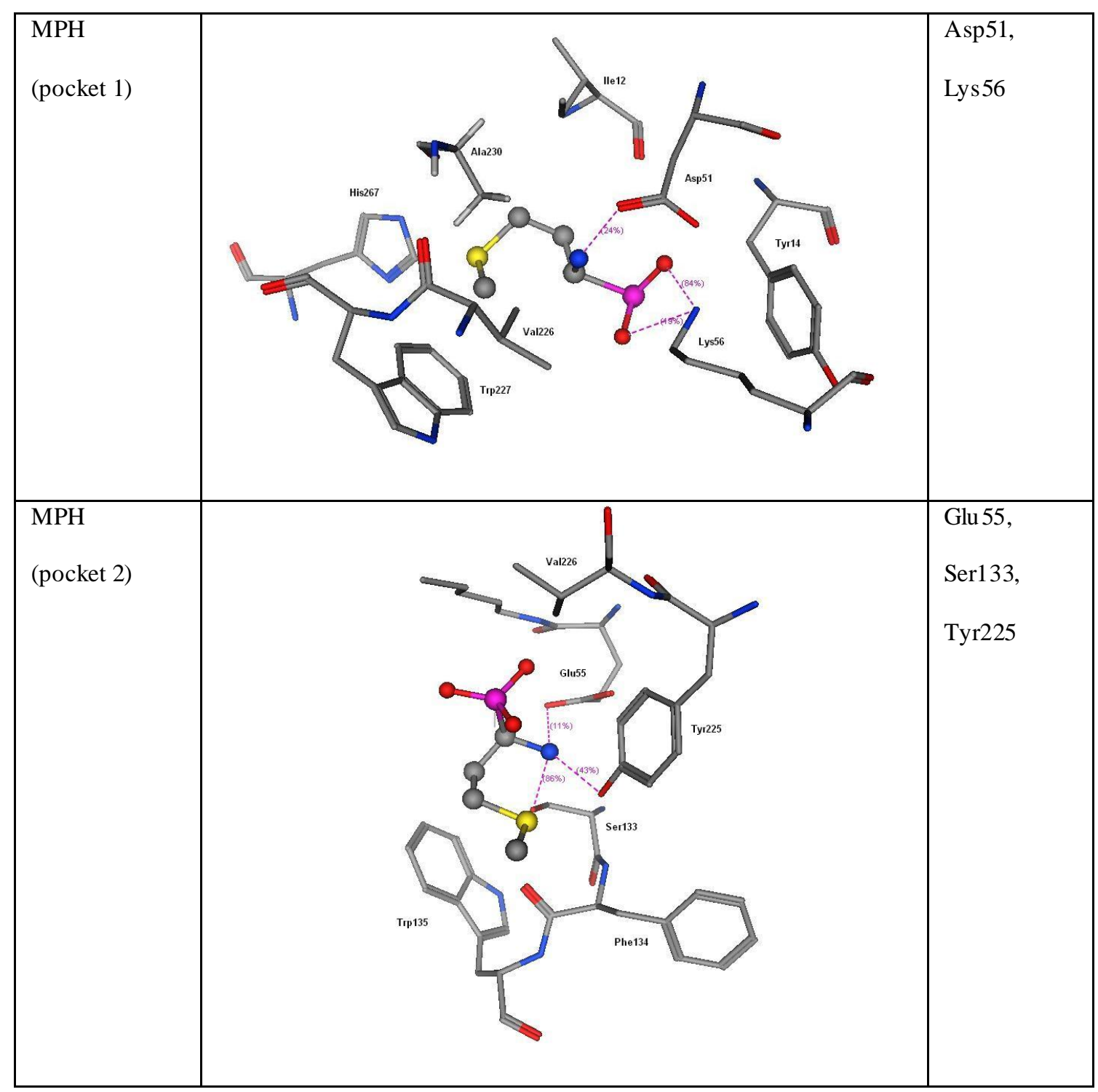




\section{Figure captions}

Fig. 1 The phylogenetic tree of $C$. difficile MetRS in relation to MetRS enzyme from other organisms. Escherichia Coli (P00959); Pseudomonas pseudomallei (Q63W90); Bovine (Q054R6); Pyrococcus Abyssi (Q9V011); Mycobacterium tuberculosis (O05593); Mycobacterium smegmatis (A0R3E2); Saccharomyces cerevisiae (Baker's yeast) (P22438); Candida albicans (O74634); Drosophila metanogaster (Fruit fly) (Q9VFL5); African clawed frog (Q7T0Z0); Japanese pufferfish (Q90YI3); Human (Q96GW9); Mouse (Q499X9); Streptococcus pneumoniae (P67580); Stapphylococcus aureus (Q5H116); Clostridium difficile (Q181D9); Clostridium acetobutylicum (Q97EW5); Thermus thermophilus (P23395); Aquifex aeolicus (O67298); Camphylobacter jejuni (Q9PP85); Helicobacter pylori (P56127)

Fig. 2 Sequence alignment of MetRS enzymes of Aquifex aeolicus, Clostridium difficile, Thermus thermophilus, Pyrococcus Abyssi and Escherichia Coli (E) using Clustal W2 in which "*" means that the residues are identical, ":" means that conserved substitutions have been observed, "." means that semi-conserved substitutions are observed. The residues are colored according to their chemical properties where red, small hydrophobic (AVFPMILWY); blue, acidic (DE); purple, basic (RHK); green, hydroxyl + amine + basic (STYHCNGQ)

Fig. 3 Superimposition of 2CSX (purple) and 1A8H (green)

Fig. 4 Superimposition of C.difficile model residues (cyan, $\mathrm{Cd}$ ) and T. thermophilus zinc bindng template $(1 \mathrm{~A} 8 \mathrm{H}$, grey, Aa) residues interacting with the zinc ion (yellow sphere $C$. difficile model; orange sphere $T$. thermophilus template)

Fig. 5 A. A. aeolicus Met tRNA structure [22]. B. predicted secondary structure of the C. difficile Met tRNA

Fig. 6 Met-tRNA anticodon interactions with the $C$. difficile homology model, corresponding key residues identified by analyzing homologous MetRS are highlighted in red 
Fig. 7 (a) Final $C$. difficile Met-tRNA homology model with the zinc shown as an orange sphere (top right), tRNA (left) and protein (right). (b) Model with domains indicated: Rossmann fold (orange); connective polypeptide (CP) domain consisting of antiparallel $\beta$ sheet core (cyan), MetRS-specific $\beta$-strand insertion (green), zinc finger (yellow), and antiparallel $\alpha_{2}$-helical structure (blue); stem contact (SC) fold domain (red); $\alpha$-helix bundle domain (magenta)

Fig. 8 ProSA output for the $C$. difficile Met tRNA model. A. shows the local model quality by plotting energies as a function of amino acid sequence position. B. inidicated the z-score (dark spot) on a plot of z-scores of all protein chains in the PDB determined by X-ray crystallography (light blue) or NMR spectroscopy (dark blue)

Fig. 9 Representative REP3123 docking into C. difficile MetRS model showing Gaussian molecular surface contacts. H-bonding, Hydrophobic, Mild polar 
A homology model for Clostridium difficile methi onyl tRNA synthetase: active site analysis and docking interactions

Ehab Al-Moubarak and Claire Simons
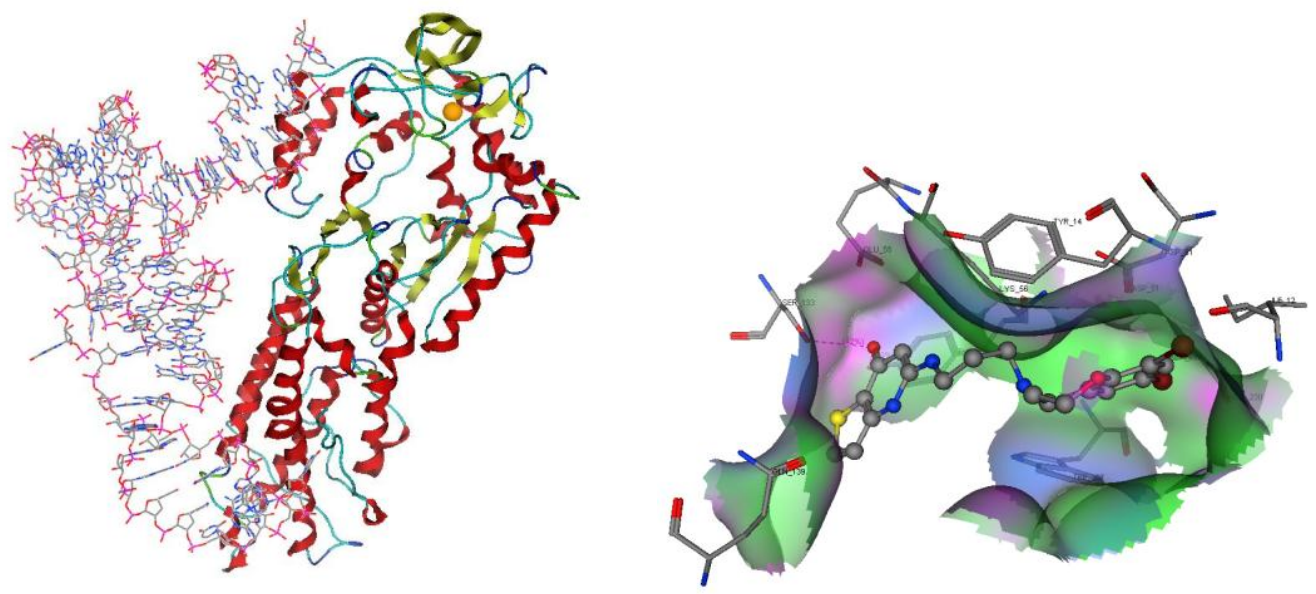

Using homology modeling the active site domain of $C$. difficile MetRS and key binding interactions have been identified, providing a tool for drug design and the development of inhibitors with greater selectivity. 